\title{
QUBITS AS SPECTROMETERS OF QUANTUM NOISE
}

\author{
R.J. SCHOELKOPF, A.A. CLERK, S.M. GIRVIN, K.W. LEHN- \\ ERT and M.H. DEVORET \\ Departments of Applied Physics and Physics \\ Yale University, PO Box 208284, New Haven, CT 06520-8284
}

\section{Introduction}

Electrical engineers and physicists are naturally very interested in the noise of circuits, amplifiers and detectors. This noise has many origins, some of which are completely unavoidable. For example, a dissipative element (a resistor) at finite temperature inevitably generates Johnson noise. Engineers long ago developed spectrum analyzers to measure the intensity of this noise. Roughly speaking, these spectrum analyzers consist of a resonant circuit to select a particular frequency of interest, followed by an amplifier and square law detector (e.g. a diode rectifier) which measures the mean square amplitude of the signal at that frequency.

With the advent of very high frequency electronics operating at low temperatures, we have entered a new regime $\hbar \omega>k_{\mathrm{B}} T$, where quantum mechanics plays an important role and one has to begin to think about quantum noise and quantum-limited amplifiers and detectors. This topic is well-studied in the quantum optics community and is also commonplace in the radio astronomy community. It has recently become of importance in connection with quantum computation and the construction of mesoscopic electrical circuits which act like artificial atoms with quantized energy levels. It is also important for understanding the quantum measurement process in mesoscopic systems.

In a classical picture, the intensity of Johnson noise from a resistor vanishes linearly with temperature because thermal fluctuations of the charge carriers cease at zero temperature. One knows from quantum mechanics, however, that there are quantum fluctuations even at zero temperature, due to zero-point motion. Zero-point motion is a notion from quantum mechanics that is frequently misunderstood. One might wonder, for example, whether it is physically possible to use a spectrum analyzer to detect the zero-point motion. The answer is quite definitely yes, if we use a quantum 
system! Consider for example a hydrogen atom in the $2 \mathrm{p}$ excited state lying $3 / 4$ of a Rydberg above the 1 s ground state. We know that this state is unstable and has a lifetime of only about 1 ns before it decays to the ground state and emits an ultraviolet photon. This spontaneous decay is a natural consequence of the zero-point motion of the electromagnetic fields in the vacuum surrounding the atom. In fact, the rate of spontaneous decay gives a simple way in which to measure this zero point motion of the vacuum. Placing the atom in a resonant cavity can modify the strength of the noise at the transition frequency, and this effect can be measured via a change in the decay rate.

At finite temperature, the vacuum will contain blackbody photons which will increase the rate of decay due to stimulated emission and also cause transitions in the reverse direction, $1 \mathrm{~s} \rightarrow 2 \mathrm{p}$, by photon absorption. With these ideas in mind, it is now possible to see how to build a quantum spectrum analyzer.

The remainder of this article is organized as follows. First we describe the general concept of a two-level system as a quantum spectrum analyzer. We next review the Caldeira-Leggett formalism for the modelling of a dissipative circuit element, such as a resistor, and its associated quantum noise. Then, a brief discussion of the single Cooper-pair box, a circuit which behaves as a two-level system or qubit, is given. We then discuss the effects of a dissipative electromagnetic environment on the box, and treat the case of a simple linear, but nonequilibrium environment, consisting of a classical tunnel junction which produces shot noise under bias. Finally, we describe a theoretical technique for calculating the properties of a Cooper-pair box coupled to a measurement system, which will be a nonlinear, nonequilibrium device, such as a single-electron transistor. Equivalently, this allows one to calculate the full quantum noise spectrum of the measurement device. Results of this calculation for the case of a normal SET are presented.

\section{Two-level systems as spectrum analyzers}

Consider a quantum system (atom or electrical circuit) which has its two lowest energy levels $\epsilon_{0}$ and $\epsilon_{1}$ separated by energy $E_{01}=\hbar \omega_{01}$. We suppose for simplicity that all the other levels are far away in energy and can be ignored. The states of any two-level system can be mapped onto the states of a fictitious spin- $1 / 2$ particle since such a spin also has only two states in its Hilbert space. With spin up representing the ground state $(|g\rangle)$ and spin down representing the excited state $(|e\rangle)$, the Hamiltonian is (taking the zero of energy to be the center of gravity of the two levels)

$$
H_{0}=-\frac{\hbar \omega_{01}}{2} \sigma_{z}
$$


In keeping with the discussion above, our goal is to see how the rate of 'spinflip' transitions induced by an external noise source can be used to analyze the spectrum of that noise. Suppose for example that there is a noise source with amplitude $f(t)$ which can cause transitions via the perturbation ${ }^{1}$

$$
V=A f(t) \sigma_{x}
$$

where $A$ is a coupling constant. The variable $f(t)$ represents the noise source. We can temporarily pretend that $f$ is a classical variable, although its quantum operator properties will be forced upon us very soon. For now, only our two-level spectrum analyzer will be treated quantum mechanically.

We assume that the coupling $A$ is under our control and can be made small enough that the noise can be treated in lowest order perturbation theory. We take the state of the two-level system to be

$$
|\psi(t)\rangle=\left(\begin{array}{c}
\alpha_{g}(t) \\
\alpha_{e}(t)
\end{array}\right)
$$

In the interaction representation, first-order time-dependent perturbation theory gives

$$
\left|\psi_{\mathrm{I}}(t)\right\rangle=|\psi(0)\rangle-\frac{i}{\hbar} \int_{0}^{t} d \tau \hat{V}(\tau)|\psi(0)\rangle .
$$

If we initially prepare the two-level system in its ground state then the amplitude to find it in the excited state at time $t$ is

$$
\begin{aligned}
\alpha_{e} & =-\frac{i A}{\hbar} \int_{0}^{t} d \tau\left\langle e\left|\hat{\sigma}_{x}(\tau)\right| g\right\rangle f(\tau)+O\left(A^{2}\right), \\
& =-\frac{i A}{\hbar} \int_{0}^{t} d \tau e^{i \omega_{01} \tau} f(\tau)+O\left(A^{2}\right) .
\end{aligned}
$$

We can now compute the probability

$$
p_{e}(t) \equiv\left|\alpha_{e}\right|^{2}=\frac{A^{2}}{\hbar^{2}} \int_{0}^{t} \int_{0}^{t} d \tau_{1} d \tau_{2} e^{-i \omega_{01}\left(\tau_{1}-\tau_{2}\right)} f\left(\tau_{1}\right) f\left(\tau_{2}\right)+O\left(A^{3}\right)
$$

We are actually only interested on the average time evolution of the system

$$
\bar{p}_{e}(t)=\frac{A^{2}}{\hbar^{2}} \int_{0}^{t} \int_{0}^{t} d \tau_{1} d \tau_{2} e^{-i \omega_{01}\left(\tau_{1}-\tau_{2}\right)}\left\langle f\left(\tau_{1}\right) f\left(\tau_{2}\right)\right\rangle+O\left(A^{3}\right)
$$

\footnotetext{
1 The most general perturbation would also couple to $\sigma_{y}$ but we assume that (as is often, though not always, the case) a spin coordinate system can be chosen so that the perturbation only couples to $\sigma_{x}$. Noise coupled to $\sigma_{z}$ commutes with the Hamiltonian but is nevertheless important in dephasing coherent superpositions of the two states. We will not discuss such processes here.
} 
We can now perform a change of variables in the integrals, $\tau=\tau_{1}-\tau_{2}$ and $T=\left(\tau_{1}+\tau_{2}\right) / 2$, and we get

$$
\bar{p}_{e}(t)=\frac{A^{2}}{\hbar^{2}} \int_{0}^{t} d T \int_{-B(T)}^{B(T)} d \tau e^{-i \omega_{01} \tau}\langle f(T+\tau / 2) f(T-\tau / 2)\rangle+O\left(A^{3}\right)
$$

where

$$
\begin{aligned}
B(T) & =T \text { if } T<t / 2 \\
& =t-T \text { if } T>t / 2 .
\end{aligned}
$$

Let us now suppose that the noise correlation function is stationary (time translation invariant) and has a finite but small autocorrelation time $\tau_{f}$. Then for $t \gg \tau_{f}$ we can set the bound $B(T)$ to infinity in the last integral and write

$$
\bar{p}_{e}(t)=\frac{A^{2}}{\hbar^{2}} \int_{0}^{t} d T \int_{-\infty}^{\infty} d \tau e^{-i \omega_{01} \tau}\langle f(\tau) f(0)\rangle+O\left(A^{3}\right)
$$

The integral over $\tau$ is effectively a sum of a very large number $N \sim t / \tau_{f}$ of random terms ${ }^{2}$ and hence the value undergoes a random walk as a function of time. Introducing the noise spectral density

$$
S_{f}(\omega)=\int_{-\infty}^{+\infty} d \tau e^{i \omega \tau}\langle f(\tau) f(0)\rangle
$$

we find that the probability to be in the excited state increases linearly with time, ${ }^{3}$

$$
\bar{p}_{e}(t)=t \frac{A^{2}}{\hbar^{2}} S_{f}\left(-\omega_{01}\right)
$$

The time derivative of the probability gives the transition rate

$$
\Gamma_{\uparrow}=\frac{A^{2}}{\hbar^{2}} S_{f}\left(-\omega_{01}\right)
$$

Note that we are taking in this last expression the spectral density on the negative frequency side. If $f$ were a strictly classical source $\langle f(\tau) f(0)\rangle$ would

\footnotetext{
${ }^{2}$ The size of these random terms depends on the variance of $f$ and on the value of $\omega_{01} \tau_{f}$ For $\omega_{01} \tau_{f} \gg 1$ the size will be strongly reduced by the rapid phase oscillations of the exponential in the integrand.

3 Note that for very long times, where there is a significant depletion of the probability of being in the initial state, first-order perturbation theory becomes invalid. However, for sufficiently small $A$, there is a wide range of times $\tau_{f} \ll t \ll 1 / \Gamma$ for which Eq. 12 is valid. Eqs. 13 and 14 then yield well-defined rates which can be used in a master equation to describe the full dynamics including long times.
} 
be real and $S_{f}\left(-\omega_{01}\right)=S_{f}\left(+\omega_{01}\right)$. However, because as we discuss below $f$ is actually an operator acting on the environmental degrees of freedom, $[f(\tau), f(0)] \neq 0$ and $S_{f}\left(-\omega_{01}\right) \neq S_{f}\left(+\omega_{01}\right)$.

Another possible experiment is to prepare the two-level system in its excited state and look at the rate of decay into the ground state. The algebra is identical to that above except that the sign of the frequency is reversed:

$$
\Gamma_{\downarrow}=\frac{A^{2}}{\hbar^{2}} S_{f}\left(+\omega_{01}\right)
$$

We now see that our two-level system does indeed act as a quantum spectrum analyzer for the noise. Operationally, we prepare the system either in its ground state or in its excited state, weakly couple it to the noise source, and after an appropriate interval of time (satisfying the above inequalities) simply measure whether the system is now in its excited state or ground state. Repeating this protocol over and over again, we can find the probability of making a transition, and thereby infer the rate and hence the noise spectral density at positive and negative frequencies. Note that in contrast with a classical spectrum analyzer, we can separate the noise spectral density at positive and negative frequencies from each other since we can separately measure the downward and upward transition rates. Negative frequency noise transfers energy from the noise source to the spectrometer. That is, it represents energy emitted by the noise source. Positive frequency noise transfers energy from the spectrometer to the noise source. ${ }^{4}$ In order to exhibit frequency resolution, $\Delta \omega$, adequate to distinguish these two cases, it is crucial that the two-level quantum spectrometer have sufficient phase coherence so that the linewidth of the transitions satisfies the condition $\omega_{01} / \Delta \omega \geq \max \left[k_{\mathrm{B}} T / \hbar \omega_{01}, 1\right]$.

In thermodynamic equilibrium, the transition rates must obey detailed balance $\Gamma_{\downarrow} / \Gamma_{\uparrow}=e^{\beta \hbar \omega_{01}}$ in order to give the correct equilibrium occupancies of the two states of the spectrometer. This implies that the spectral densities obey

$$
S_{f}\left(+\omega_{01}\right)=e^{\beta \hbar \omega_{01}} S_{f}\left(-\omega_{01}\right) .
$$

Without the crucial distinction between positive and negative frequencies, and the resulting difference in rates, one always finds that our two level

\footnotetext{
4 Unfortunately, there are several conventions in existence for describing the noise spectral density. It is common in engineering contexts to use the phrase 'spectral density' to mean $S_{f}(+\omega)+S_{f}(-\omega)$. This is convenient in classical problems where the two are equal. In quantum contexts, one sometimes sees the asymmetric part of the noise $S_{f}(+\omega)-S_{f}(-\omega)$ referred to as the 'quantum noise.' We feel it is simpler and clearer to simply discuss the spectral density for positive and negative frequencies separately, since they have simple physical interpretations and directly relate to measurable quantities. This convention is especially useful in non-equilibrium situations where there is no simple relation between the spectral densities at positive and negative frequencies.
} 
systems are completely unpolarized. If, however, the noise source is an amplifier or detector biased to be out of equilibrium, no general relation holds.

We now rigorously treat the quantity $f(\tau)$ as quantum operator in the Hilbert space of the noise source. The previous derivation is unchanged, and Eqs. $(13,14)$ are still valid provided that we interpret the angular brackets in Eq. (8) as representing the quantum statistical expectation value for the operator correlation (in the absence of the coupling to the spectrometer)

$$
S_{f}(\omega)=\int_{-\infty}^{+\infty} d \tau e^{i \omega \tau} \sum_{\alpha, \gamma} \rho_{\alpha \alpha}\langle\alpha|f(\tau)| \gamma\rangle\langle\gamma|f(0)| \alpha\rangle
$$

where for simplicity we have assumed that (in the absence of the coupling to the spectrometer) the density matrix is diagonal in the energy eigenbasis and time-independent (but not necessarily given by the equilibrium expression). This yields the standard quantum mechanical expression for the spectral density

$$
\begin{aligned}
S_{f}(\omega) & =\int_{-\infty}^{+\infty} d \tau e^{i \omega \tau} \sum_{\alpha, \gamma} \rho_{\alpha \alpha} e^{\frac{i}{\hbar}\left(\epsilon_{\alpha}-\epsilon_{\gamma}\right) t}|\langle\alpha|f| \gamma\rangle|^{2} \\
& =2 \pi \hbar \sum_{\alpha, \gamma} \rho_{\alpha \alpha}|\langle\alpha|f| \gamma\rangle|^{2} \delta\left(\epsilon_{\gamma}-\epsilon_{\alpha}-\hbar \omega\right) .
\end{aligned}
$$

Substitution of this into Eqs. $(13,14)$ we derive the familiar Fermi Golden Rule expressions for the two transition rates.

In standard courses, one is not normally taught that the transition rate of a discrete state into a continuum as described by Fermi's Golden Rule can (and indeed should!) be viewed as resulting from the continuum acting as a quantum noise source. The above derivation hopefully provides a motivation for this interpretation.

One standard model for the continuum is an infinite collection of harmonic oscillators. The electromagnetic continuum in the hydrogen atom case mentioned above is a prototypical example. The vacuum electric field noise coupling to the hydrogen atom has an extremely short autocorrelation time because the range of mode frequencies $\omega_{\alpha}$ (over which the dipole matrix element coupling the atom to the mode electric field $\vec{E}_{\alpha}$ is significant) is extremely large, ranging from many times smaller than the transition frequency to many times larger. Thus the autocorrelation time of the vacuum electric field noise is considerably less than $10^{-15} \mathrm{~s}$, whereas the decay time of the hydrogen $2 \mathrm{p}$ state is about $10^{-9} \mathrm{~s}$. Hence the inequalities needed for the validity of our expressions are very easily satisfied.

Of course in the final expression for the transition rate, energy conservation means that only the spectral density at the transition frequency 
enters. However, in order for the expression to be valid (and in order for the transition rate to be time independent), it is essential that there be a wide range of available photon frequencies so that the vacuum noise has an autocorrelation time much shorter than the inverse of the transition rate.

\section{Quantum Noise from a Resistor}

Instead of an atom in free space, we might consider a quantum bit capacitively coupled to a transmission line. The transmission line is characterized by an inductance per unit length $\ell$ and capacitance per unit length $c$. A semi-infinite transmission line presents a frequency-independent impedance $Z=R_{0}=\sqrt{\ell / c}$ at its end and hence acts like an ideal resistor. The dissipation is caused by the fact that currents injected at one end launch waves which travel off to infinity and do not return. Very conveniently, however, the system is simply a large collection of harmonic oscillators (the normal modes) and hence can be readily quantized. This representation of a physical resistor is essentially the one used by Caldeira and Leggett [1] in their seminal studies of the effects of dissipation on tunneling. The only difference between this model and the vacuum fluctuations in free space discussed above is that the relativistic bosons travel in one dimension and do not carry a polarization label. This changes the density of states as a function of frequency, but has no other essential effect.

The Lagrangian for the system is

$$
\mathcal{L}=\int_{0}^{\infty} d x \frac{\ell}{2} j^{2}-\frac{1}{2 c} q^{2}
$$

where $j(x, t)$ is the local current density and $q(x, t)$ is the local charge density. Charge conservation connects these two quantities via the constraint

$$
\partial_{x} j(x, t)+\partial_{t} q(x, t)=0 .
$$

We can solve this constraint by defining a new variable

$$
\theta(x, t) \equiv \int_{0}^{x} d x^{\prime} q\left(x^{\prime}, t\right)
$$

in terms of which the current density is $j(x, t)=-\partial_{t} \theta(x, t)$ and the charge density is $q(x, t)=\partial_{x} \theta(x, t)$. For any well-behaved function $\theta(x, t)$, the continuity equation is automatically satisfied so there are no dynamical constraints on the $\theta$ field. In terms of this field the Lagrangian becomes

$$
\mathcal{L}=\int_{0}^{\infty} d x \frac{\ell}{2}\left(\partial_{t} \theta\right)^{2}-\frac{1}{2 c}\left(\partial_{x} \theta\right)^{2}
$$


The Euler-Lagrange equation for this Lagrangian is simply the wave equation $v^{2} \partial_{x}^{2} \theta-\partial_{t}^{2} \theta=0$ where the mode velocity is $v=1 / \sqrt{\ell c}$.

¿From Eq. (21) we can deduce that the proper boundary conditions (in the absence of any coupling to the qubit) for the $\theta$ field are $\theta(0, t)=$ $\theta(L, t)=0$. (We have temporarily made the transmission line have a finite length $L$.) The normal mode expansion that satisfies these boundary conditions is

$$
\theta(x, t)=\sqrt{\frac{2}{L}} \sum_{n=1}^{\infty} \varphi_{n}(t) \sin \frac{k_{n} \pi x}{L},
$$

where $\varphi_{n}$ is the normal coordinate and $k_{n} \equiv \frac{\pi n}{L}$. Substitution of this form into the Lagrangian and carrying out the spatial integration yields a set of independent harmonic oscillators representing the normal modes.

$$
\mathcal{L}=\sum_{n=1}^{\infty} \frac{\ell}{2}\left(\dot{\varphi}_{n}\right)^{2}-\frac{1}{2 c} k_{n}^{2} \varphi_{n}^{2}
$$

From this we can find the momentum $p_{n}$ canonically conjugate to $\varphi_{n}$ and quantize the system to obtain an expression for the voltage at the end of the transmission line in terms of the mode creation and destruction operators

$$
V=\sqrt{\frac{2}{L}} \frac{1}{c} \partial_{x} \theta(0, t)=\frac{1}{c} \sum_{n=1}^{\infty} k_{n} \sqrt{\frac{\hbar}{2 \ell \Omega_{n}}}\left(a_{n}^{\dagger}+a_{n}\right) .
$$

The spectral density of voltage fluctuations is then found to be

$$
S_{V}(\omega)=2 \pi \frac{2}{L} \sum_{n=1}^{\infty} \frac{\hbar \Omega_{n}}{2 c}\left\{n_{\gamma}\left(\hbar \Omega_{n}\right) \delta\left(\omega+\Omega_{n}\right)+\left[n_{\gamma}\left(\hbar \Omega_{n}\right)+1\right] \delta\left(\omega-\Omega_{n}\right)\right\},
$$

where $n_{\gamma}(\hbar \omega)$ is the Bose occupancy factor for a photon with energy $\hbar \omega$. Taking the limit $L \rightarrow \infty$ and converting the summation to an integral yields

$$
S_{V}(\omega)=2 R_{0} \hbar|\omega|\left\{n_{\gamma}(\hbar|\omega|) \Theta(-\omega)+\left[n_{\gamma}(\hbar \omega)+1\right] \Theta(\omega)\right\},
$$

where $\Theta$ is the step function. We see immediately that at zero temperature there is no noise at negative frequencies because energy can not be extracted from zero-point motion. However there remains noise at positive frequencies indicating that the vacuum is capable of absorbing energy from the qubit.

A more compact expression for this 'two-sided' spectral density of a resistor is

$$
S_{V}(\omega)=\frac{2 R_{0} \hbar \omega}{1-e^{-\hbar \omega / k_{\mathrm{B}} T}}
$$

which reduces to the more familiar expressions in various limits. For example, in the classical limit $k_{\mathrm{B}} T \gg \hbar \omega$ the spectral density is equal to the 
Johnson noise result ${ }^{5}$

$$
S_{V}(\omega)=2 R_{0} k_{\mathrm{B}} T
$$

which is frequency independent, and in the quantum limit it reduces to

$$
S_{V}(\omega)=2 R_{0} \hbar \omega \Theta(\omega)
$$

Again, the step function tells us that the resistor can only absorb energy, not emit it, at zero temperature.

If we use the engineering convention and add the noise at positive and negative frequencies we obtain

$$
S_{V}(\omega)+S_{V}(-\omega)=2 R_{0} \hbar \omega \operatorname{coth} \frac{\hbar \omega}{2 k_{\mathrm{B}} T}
$$

for the symmetric part of the noise, which appears in the quantum fluctuationdissipation theorem[2]. The antisymmetric part of the noise is simply

$$
S_{V}(\omega)-S_{V}(-\omega)=2 R_{0} \hbar \omega
$$

This quantum treatment can also be applied to any arbitrary dissipative network[3]. If we have a more complex circuit containing capacitors and inductors, then in all of the above expressions, $R_{0}$ should be replaced by $\operatorname{Re} Z(\omega)$ where $Z(\omega)$ is the complex impedance presented to the qubit.

\section{The Single Cooper-Pair Box: a Two-Level Quantum Circuit}

The Cooper-pair box (CPB) is a simple circuit [4], consisting of a small superconducting "island", connected to a large reservoir via a single smallcapacitance Josephson junction, depicted as a box with a cross (Fig. 1). The island is charge biased by applying a voltage $\left(V_{g}\right)$ to a nearby lead, called the gate, which has a small capacitance to the island, $C_{g}$. The junction is characterized by its capacitance, $C_{j}$, and its tunnel resistance, $R_{j}$. At temperatures well below the transition temperature of the superconductor ( $T_{C} \sim 1.5 \mathrm{~K}$ for the usual $\mathrm{Al} / \mathrm{AlOx} / \mathrm{Al}$ junctions), none of the many $\left(\sim 10^{9}\right)$ quasiparticle states on the island should be thermally occupied, and the number of Cooper-pairs on the island is the only relevant degree of freedom.

We may then write the Hamiltonian for the box in terms of the states of different numbers of pairs on the island, which are eigenstates of the number operator, $\hat{n}|n\rangle=n|n\rangle$. The box Hamiltonian consists of an electrostatic term, plus a Josephson term describing the coupling of the island to the

\footnotetext{
${ }^{5}$ Note again that in the engineering convention this would be $S_{V}(\omega)=4 R_{0} k_{\mathrm{B}} T$.
} 
a)

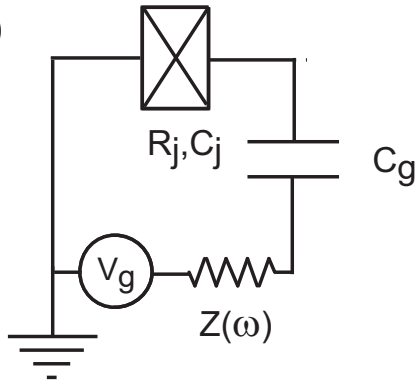

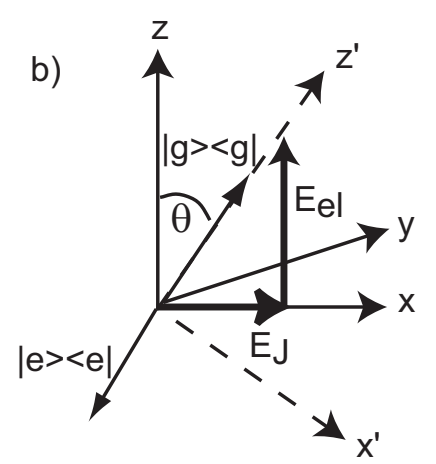

Figure 1. a) Circuit diagram of Cooper-pair box. b) Pseudo-spin representation of the energies of Cooper-pair box. The density matrix for the two pure eigenstates lie along the total effective field, collinear with the z' axis.

lead,

$$
\begin{aligned}
H & =H_{\text {electrostatic }}+H_{\text {Josephson }} \\
& =4 E_{C} \sum_{n}\left(n-n_{g}\right)^{2}|n\rangle\langle n|-\frac{E_{J}}{2} \sum_{n}(|n+1\rangle\langle n|+\text { h.c. })
\end{aligned}
$$

The energy scale for the electrostatic interaction is given by the charging energy, $E_{C}=e^{2} / 2 C_{\Sigma}$, where $C_{\Sigma}=C_{j}+C_{g}$ is the total island capacitance, while the Josephson energy, $E_{J}$, is set by the tunnel resistance and the gap of the superconductor,

$$
E_{J}=\frac{h \Delta}{8 e^{2} R_{j}}=\frac{\Delta}{8} \frac{R_{K}}{R_{j}} .
$$

The electrostatic term is easily modulated by changing the voltage on the gate; the quantity $n_{g}=C_{g} V_{g} / 2 e$ that appears in the Hamiltonian corresponds to the total polarization charge (in units of Cooper pairs) injected into the island by the voltage source.

This Hamiltonian leads to particularly simple behavior in the charge regime, when the electrostatic energy dominates over the Josephson coupling, $4 E_{C} \gg E_{J}$. In this case we can restrict the discussion to only two charge states, $|n=0\rangle$ and $|n=1\rangle$. For convenience we can reference the energies of the two states to their midpoint, $E_{m i d}=4 E_{C}\left(1-2 n_{g}\right)^{2}$, so that the Hamiltonian now becomes

$$
H=\frac{1}{2}\left(\begin{array}{cc}
-E_{e l} & -E_{J} \\
-E_{J} & E_{e l}
\end{array}\right)
$$

where $E_{e l}$ is the electrostatic energy that is now linear in the gate charge, $E_{e l}=4 E_{C}\left(1-2 n_{g}\right)$. It is also now apparent that the Hamiltonian is identical 

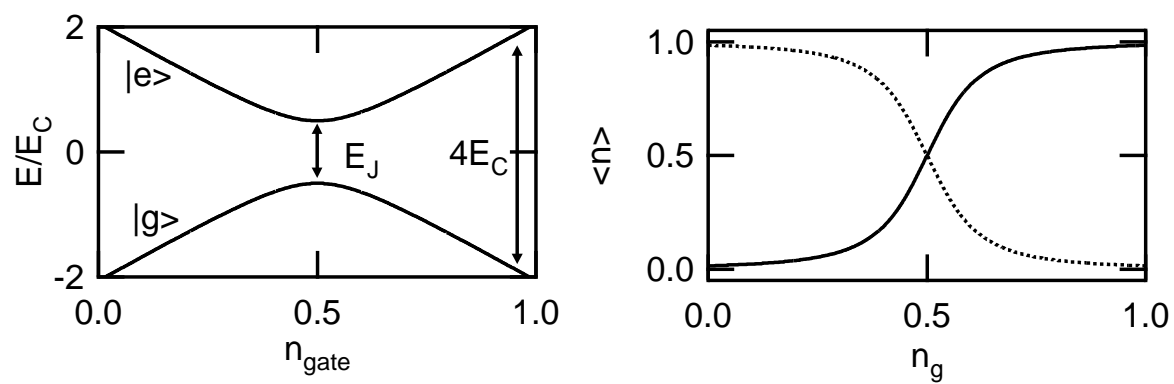

Figure 2. Energies (left) of ground and excited states of a Cooper-pair box with $E_{C}=E_{J}$ vs. dimensionless gate charge, $n_{g}=C_{g} V_{g} / 2 e$. The expectation value of a charge measurement, $\langle n\rangle$, (right) for the ground (solid line) and excited (dotted line) states vs. $n_{g}$.

to that of a fictitious spin- $1 / 2$ particle,

$$
H=-\frac{E_{e l}}{2} \sigma_{z}-\frac{E_{J}}{2} \sigma_{x}
$$

under the influence of two psuedo-magnetic fields, $B_{z}=E_{e l}$ and $B_{x}=E_{J}$, as depicted in Fig. 1. In other words, the box is a qubit or two-level system ${ }^{6}$. The state of the system is in general a linear combination of the states $|n=0\rangle$ and $|n=1\rangle$. The state can be depicted using the density matrix, which corresponds to a point on the Bloch sphere, where the north pole (+z-direction) corresponds to $|n=0\rangle$. The ground and excited states of the system will be aligned and anti-aligned with the total fictitious field, i.e. in the $\pm z^{\prime}$ directions.

It is also apparent from this discussion that the states of the box can be easily manipulated by changing the gate voltage. The energies of the ground and excited states, as a function of $n_{g}$, are displayed in Figure 2. The energy difference between the ground and excited bands varies from $4 E_{C}$ at $n_{g}=0,1$, to a minimum at the charge degeneracy point, $n_{g}=1 / 2$. At this point, the Josephson coupling leads to an avoided crossing, and the splitting is $E_{J}$.

Also plotted is the expectation value of the number operator, $\langle|\hat{n}|\rangle$, which is proportional to the total charge on the island. In the geometrical

\footnotetext{
${ }^{6}$ Of course, this is an approximation, as there are other charge states $(|n=2\rangle$, etc. $)$ which are possible, but require much higher energy. Even outside the charge regime (i.e. when $E_{J} \geq 4 E_{C}$ ) the two lowest levels of the box can be used to realize a qubit [5]. In this case, the two states do not exactly correspond with eigenstates of charge, and matrix elements are more complicated to calculate. Nonetheless, this regime can also be used as an electrical quantum spectrum analyzer.
} 
picture of Fig. 1b, a measurement of charge $(\hat{n})$ is equivalent to projecting the state on the z-axis, $\hat{n}=\frac{1}{2}\left(1-\sigma_{z}\right)$. We see that as the gate charge is changed from 0 to 1 , the ground state is initially $|n=0\rangle$, and the character of the ground and excited states interchange on passing through the degeneracy point, leading to the transition between $\langle\hat{n}\rangle=0$ and 1, which is broadened by quantum fluctuations (the $\sigma_{x}$ coupling). At the degeneracy point, the ground and excited states lie in the $\pm x$-directions, i.e. they are symmetric and antisymmetric combinations of the two charge states. In general, we will denote the ground and excited state of the $\mathrm{CPB}$ at a particular gate voltage as $|g\rangle$ and $|e\rangle$, which are given in terms of the charge states by $|g\rangle=\cos (\theta / 2)|0\rangle+\sin (\theta / 2)|1\rangle$ and $|e\rangle=-\sin (\theta / 2)|0\rangle+\cos (\theta / 2)|1\rangle$ respectively, where $\theta=\arctan \left[E_{J} / E_{e l}\right]$ is a function of the gate voltage.

A nice property of the $\mathrm{CPB}$ in this regime is that the various matrix elements can be calculated in a straightforward way. For example, the expectation value of $\hat{n}$ in the ground state, $\langle g|\hat{n}| g\rangle$, is therefore equal to $1 / 2\left(1-\left\langle g\left|\sigma_{z}\right| g\right\rangle\right)=\sin ^{2}(\theta / 2)$, from which we can find the ground state charge as shown in Fig. 2. A perturbation in the gate charge, due for example to a fluctuation or change in the applied gate voltage, will lead to a proportional change in the electrostatic energy, or the z-component of the fictitious magnetic field. Such a perturbation will cause both dephasing and transitions between states.

\section{General Discussion of CPB Coupled to a Dissipative Environment}

In the previous section we described the Hamiltonian and the eigenstates for a Cooper-pair box which is "charge-biased," i.e. controlled with a voltage applied to a gate capacitor $C_{g}$, as shown in Fig. 1. In our earlier treatment of the box, the voltage and the dimensionless gate charge, $n_{g}$ were treated as fixed parameters of the Hamiltonian (c-numbers). In this case, the box's evolution is purely deterministic and conservative. However, it is impossible, even in principle, to control such a voltage with arbitrary precision at all frequencies. In Fig. 1, the idealized source of the gate voltage is drawn in series with an impedance $Z(\omega)$ of the gate lead. Generally, this gate lead will be connected to external wiring (a transmission line), with a typical real impedance comparable to the impedance of free space $(\sim 50 \Omega)$ at the microwave transition frequencies of the box. ¿From the fluctuationdissipation theorem we know that this impedance will introduce noise on the gate voltage, even at zero temperature.

There are several effects of the voltage noise on the box, or the coupling of our spin- $1 / 2$ circuit to the many external degrees of freedom represented by the gate impedance. First, even at zero temperature, we will find a finite excited state lifetime, $T 1$, for the box. Second, at finite temperature, we will 
find a finite polarization of our psuedo-spin, i.e. some steady-state probability to find the spin in its excited state. Finally, the gate noise introduces a random effective field felt by the spin, and a loss of phase coherence for a superpostition state. It is this last effect which is most important in making high-fidelity qubits and performing quantum computations, but it is the first two which depend most explicitly on the quantum nature of the noise. We deal in this manuscript with only these first two features of the box's coupling to the electromagnetic environment, and ignore the dephasing ${ }^{7}$. Of course, the other parameter in the Hamiltonian, the Josephson energy, can in principle fluctuate, especially as in many experiments the box's junction is split into a small SQUID in order to provide external tuning of $E_{J}$ with an applied flux. We concentrate here only on the voltage noise (fluctuations in the $\sigma_{z}$ part of the Hamiltonian) for simplicity.

We begin with a very simple treatment of the dynamics of the two-level system under the influence of the gate voltage noise. We are interested in the ensemble-averaged behavior of our psuedo-spin, which is best described using the density matrix approach, and is detailed in Section 7 on the coupling of the box to a measuring SET. The basic effects, apart from dephasing, however, can be captured simply by examining the probabilities $p_{g}$ and $p_{e}$ of finding the box in its ground $(|g\rangle)$ or excited $(|e\rangle)$ states. The noise of the external environment can drive transitions from ground to excited state and vice-versa, at rates $\Gamma_{\uparrow}$ and $\Gamma_{\downarrow}$, respectively. The coupled master equations for these probabilities are

$$
\begin{aligned}
& \frac{d p_{e}}{d t}=p_{g} \Gamma_{\uparrow}-p_{e} \Gamma_{\downarrow} \\
& \frac{d p_{g}}{d t}=p_{e} \Gamma_{\downarrow}-p_{g} \Gamma_{\uparrow}
\end{aligned}
$$

Of course conservation of probability tells us that $p_{e}+p_{g}=1$, so we introduce the polarization of the spin- $1 / 2$ system, $P=p_{g}-p_{e}$. In steadystate, the detailed balance condition is $p_{e} \Gamma_{\downarrow}=p_{g} \Gamma_{\uparrow}$. The two rates $\Gamma_{\uparrow}$ and $\Gamma_{\downarrow}$ are related by Equations 13 and 14 to the spectral densities of the noise at negative and positive frequencies. We see immediately that if the spectral density is symmetric (classical!), then the rates for transitions up and down are equal, the occupancies of the two states are exactly equal, and the polarization of the psuedo-spin is identically zero. It is the quantum, or antisymmetric, part of the noise which gives the finite polarization of the spin. Even in NMR, where the temperature is large compared to the level splitting $\left(\hbar \omega_{01} \leq k_{\mathrm{B}} T\right)$, this effect is well-known and crucial, as the small but non-zero polarization is the subject of the field!

\footnotetext{
${ }^{7}$ For a nice recent treatment of dephasing in Josephson junctions, see Ref. [6].
} 
Solving for the steady-state polarization, we find

$$
P_{s s}=\frac{\Gamma_{\downarrow}-\Gamma_{\uparrow}}{\Gamma_{\downarrow}+\Gamma_{\uparrow}}=\frac{S\left(+\omega_{01}\right)-S\left(-\omega_{01}\right)}{S\left(+\omega_{01}\right)+S\left(-\omega_{01}\right)}
$$

An measurement of the steady-state polarization allows one to observe the amount of asymmetry in the noise, so the two-level system is a quantum spectrum analyzer.

If we can create a non-equilibrium polarization, $P=P_{s s}+\Delta P$ (a pure state is not necessary) of our two-level system, we expect it to return to the steady state value. Substituting the modified probabilities $p_{e}(t)=p_{e_{s s}}-$ $\Delta P(t) / 2$ and $p_{g}(t)=p_{g_{s s}}+\Delta P(t) / 2$ into our master equations above, we find an equation for the deviation of the polarization

$$
\frac{d(\Delta P(t))}{d t}=-\Delta P(t)\left(\Gamma_{\uparrow}+\Gamma_{\downarrow}\right) .
$$

Thus the system decays to its steady-state polarization with the relaxation rate $\Gamma_{1}=\Gamma_{\uparrow}+\Gamma_{\downarrow}=(A / \hbar)^{2}\left[S\left(-\omega_{01}\right)+S\left(+\omega_{01}\right)\right]$ related to the total noise at both positive and negative frequencies. In NMR, the time $1 / \Gamma_{1}$ is referred to as $T 1$. In the zero-temperature limit, there is no possibility of the qubit absorbing energy from the environment, so $\Gamma_{\uparrow}=0$, and we find full polarization $P=1$, and a decay of any excited state population at a rate $\Gamma_{\downarrow}=1 / T 1$ which is the spontaneous emission rate.

It is worth emphasizing that a quantum noise source is always characterized by two numbers (at any frequency), related to the positive and negative frequency spectral densities, or to the symmetric and antisymmetric parts of the noise. These two quantities have different effects on a two-level system, introducing a finite polarization and finite excited-state lifetime. Consequently, a measurement of both the polarization and $T 1$ of a two-level system is needed to fully characterize the quantum noise coupled to the qubit. Such measurements in electrical systems are now possible, and some of us [7] have recently performed such a characterization for the specific case of a CPB coupled to a superconducting single-electron transistor.

Our discussion in this section uses the language of NMR to describe the effects on the two-level system. There are, however, several possible protocols ${ }^{8}$ for measuring the quantum noise, and several different "basis sets" or measured quantities which describe the noise process or the quantum reservoir to which the two-level system is coupled. Table 1 contains a "translation" between the specific pairs of quantities that are commonly

${ }^{8}$ The idea of watching the decay from the pure states $|e\rangle$ and $|g\rangle$ to measure $S_{V}\left( \pm\left|\omega_{01}\right|\right)$ separately was described in Section 2 .

rjsdraft12.tex; 28/05/2018; 5:41; p.14 
TABLE I. Different ways to characterize a quantum reservoir.

\begin{tabular}{lcc}
\hline Fermi Golden Rule & $\Gamma_{\uparrow}(\omega)=\frac{A^{2}}{\hbar^{2}} S_{V}(+|\omega|)$ & $\Gamma_{\downarrow}(\omega)=\frac{A^{2}}{\hbar^{2}} S_{V}(-|\omega|)$ \\
\hline Fluct.-Diss. Relation & $n_{\gamma}(\omega)=2 \Gamma_{\uparrow} /\left(\Gamma_{\downarrow}-\Gamma_{\uparrow}\right)$ & $\operatorname{Re}[Z(\omega)]=\frac{\hbar}{A^{2} \omega}\left(\Gamma_{\downarrow}-\Gamma_{\uparrow}\right)$ \\
\hline NMR & $T 1=\left(\Gamma_{\downarrow}+\Gamma_{\uparrow}\right)^{-1}$ & $P=\left(\Gamma_{\downarrow}-\Gamma_{\uparrow}\right) /\left(\Gamma_{\downarrow}+\Gamma_{\uparrow}\right)$ \\
\hline Quantum Optics & $B_{\text {Einstein }}=\Gamma_{\uparrow}$ & $A_{\text {Einstein }}=\Gamma_{\downarrow}-\Gamma_{\uparrow}$ \\
\hline
\end{tabular}

used in different disciplines, and their relation to the positive and negative noise spectral densities. In all cases, though, two separate numbers are required to specify the properties of a quantum reservoir.

\section{The Box Coupled to an Ohmic Environment}

We can now proceed to the effects of a specific dissipative coupling to the Cooper-pair box. The noise on the gate voltage will lead to a fluctuation of the gate charge parameter, $n_{g}$, and thus to a fluctuation of the electrostatic energy, i.e. the $\sigma_{z}$ term in the Hamiltonian (Eq. 37). Depending on the average value of $n_{g}$, this fluctuation will consist of fluctuations which are both longitudinal ( $\|$ to $\sigma_{z}^{\prime}$ ) and transverse $\left(\perp\right.$ to $\left.\sigma_{z}^{\prime}\right)$. To calculate the rates of transitions between the states $|e\rangle$ and $|g\rangle$, we need to find the coupling strength $A$ of this perturbation in the $\sigma_{x}^{\prime}$ direction. Referring to Fig. 1, we see that $\sigma_{z}=\cos (\theta) \sigma_{z}^{\prime}-\sin (\theta) \sigma_{x}^{\prime}$. If we let the gate charge now be $n_{g}(t)=\overline{n_{g}}+\delta n_{g}(t)$, we may rewrite the Hamiltonian Eq. 37 in the new eigenbasis as

$$
H=-\frac{E_{01}}{2 \hbar} \sigma_{z}^{\prime}+4 E_{C} \cos (\theta) \delta n_{g}(t) \sigma_{z}^{\prime}-4 E_{C} \sin (\theta) \delta n_{g}(t) \sigma_{x}^{\prime} .
$$

The time-varying term in the $\sigma_{z}^{\prime}$ direction will effectively modulate the transition frequency, $\omega_{01}=E_{01} / \hbar$, and cause dephasing. In terms of the gate voltage noise, $V(t)$, the $\sigma_{x}^{\prime}$ perturbation term has the form $A V(t) \sigma_{x}^{\prime}=$ $e \kappa \sin (\theta) V(t) \sigma_{x}^{\prime}$, where $e$ is the electron's charge and $\kappa=C_{g} / C_{\Sigma}$ is the capacitive coupling. Using Eq. 14, we find

$$
\Gamma_{\downarrow}=\left(\frac{e}{\hbar}\right)^{2} \kappa^{2} \sin ^{2}(\theta) S_{V}\left(+\omega_{01}\right)
$$

If the environment is effectively at zero temperature $\left(\hbar \omega_{01} \gg k_{\mathrm{B}} T\right)$, then $S_{V}\left(+\omega_{01}\right)=2 \hbar \omega_{01} R$, and the quality factor of the transition is

$$
Q=\omega_{01} / \Gamma_{\downarrow}=\frac{1}{\kappa^{2} \sin ^{2} \theta} \frac{R_{K}}{4 \pi R},
$$


where $R_{K}=h / e^{2}$ is the resistance quantum.

For a finite temperature, we have rates in both directions, and the polarization of the psuedo-spin is given by the ratio of the antisymmetric (Eq. 32) to symmetric (Eq. 31) spectral densities, $P=\tanh \left(\hbar \omega_{01} / 2 k_{\mathrm{B}} T\right)$, as one expects for any two-level system at temperature $T$. An example of the average charge state of a Cooper-pair box at finite temperature, and of the polarization and equilibration time T1, are shown in Figure 3. As the gate voltage is varied, the transition frequency of the box changes from a maximum near $n_{g}=0$, to a minimum $\omega_{01}=E_{J} / \hbar$ at the degeneracy point $n_{g}=0.5$ and then back again. We see that the states of the box are generally most "fragile" near the avoided crossing. First, the energy splitting is a minimum here, leading to the lowest polarization of the psuedo-spin. Second, because the eigenstates $|g\rangle$ and $|e\rangle$ point in the $\sigma_{x}$ direction, the matrix elements for the voltage fluctuations of the environment are maximal, i.e. the noise is orthogonal to the spin. This also implies that the dephasing effects are minimal at this degeneracy point, which offers great advantages for improving the decoherence times [5], but the excited state lifetimes are smallest at this point. One also sees that the lifetimes become large away from the degeneracy, where the electrostatic energy dominates over the Josephson energy, which offers advantages when measuring the charge state. In the limit that $E_{J}$ could be suppressed to zero during a measurement, the matrix elements (for voltage noise) vanish, and a quantum non-demolition (QND) measurement [8] could be performed. The idea of using the qubit as a quantum spectrum analyzer is precisely the reverse, where we measure the "destruction" in the two-level system (i.e. inelastic transitions caused by the coupling of the states to the environment), in order to learn about the quantum noise spectrum of the fluctuations.

The Cooper-pair box can of course also be used to measure the more interesting spectral densities of nonequilibrium devices. The simplest example is to replace the gate resistance by a tunnel junction. If we arrange to bias the junction using, e.g. an inductor, a dc current $I$ and an average dc voltage $V$ can be maintained across the junction. Classically, the current noise of such a tunneling process is frequency independent, $S_{I}=2 e I$. The voltage noise density presented to the CPB's gate would then be $S_{V}=2 e I R_{T}^{2}$, where $R_{T}$ is the junction tunnel resistance. In fact, this "white" spectral density can only extend up to frequencies of order $\omega=\mathrm{eV} / \hbar$, the maximum energy of electrons tunneling through the junction. The correct high-frequency form of the symmetrized noise density was calculated by Rogovin and Scalapino [9],

$$
S_{V}(\omega)=R(\hbar \omega+e V) \operatorname{coth}\left[\frac{\hbar \omega+e V}{2 k_{B} T}\right]+R(\hbar \omega-e V) \operatorname{coth}\left[\frac{\hbar \omega-e V}{2 k_{B} T}\right],
$$

rjsdraft12.tex; 28/05/2018; 5:41; p.16 

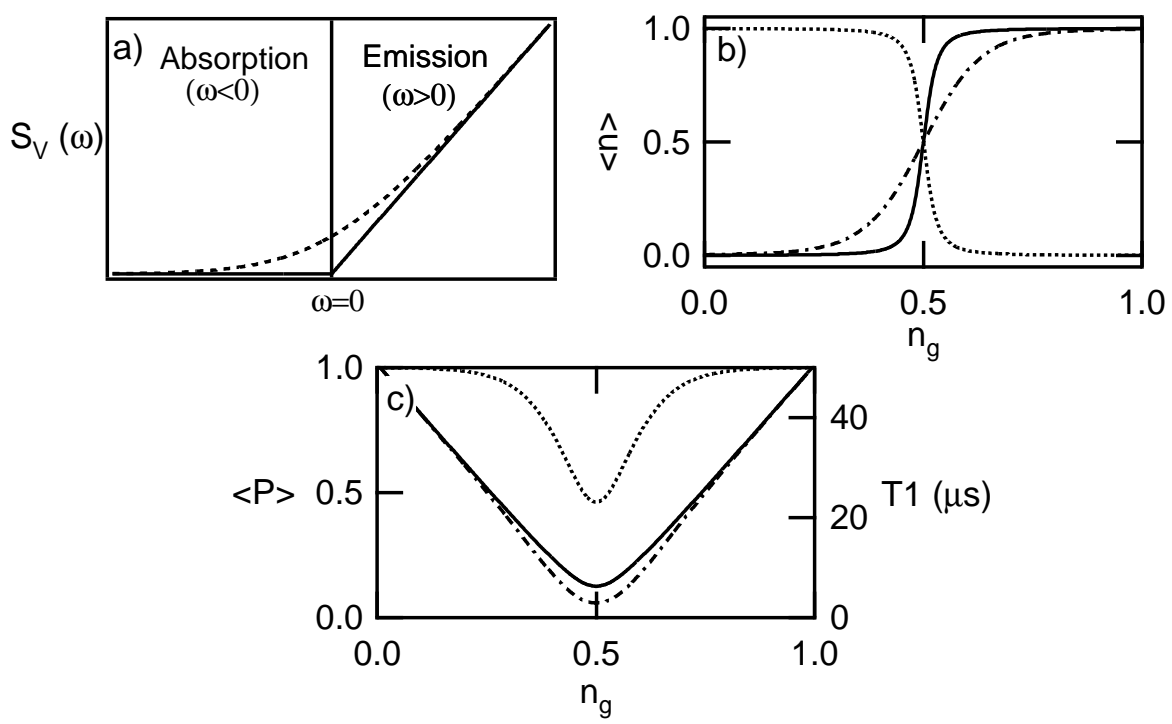

Figure 3. The box coupled to an equilibrium, Ohmic environment, i.e. a resistor. a) Two-sided noise spectral density, of the voltage, $S_{V}(\omega)$, for a resistor at $\mathrm{T}=0$ (solid line) and finite temperature (dashed line) b) Average charge of box with $E_{C}=1 K$, $E_{J}=0.5 \mathrm{~K}$ when coupled with strength $\kappa=0.01$ to a resistor with resistance $R=50 \Omega$ and temperature $T=0.5 \mathrm{~K}$. c) Polarization (dotted line) and relaxation time $\mathrm{T} 1 \mathrm{for}$ the same parameters. Full line is the rate of spontaneous emission, i.e. T1 at zero temperature.

and was indirectly measured in a mesoscopic conductor using a conventional spectrum analyzer [10]. This noise can also be expressed [11] in its two-sided form

$$
S_{V}(\omega)=\frac{(\hbar \omega+e V) R_{T}}{1-e^{-\frac{\hbar \omega+e V}{k_{B} T}}}+\frac{(\hbar \omega-e V) R_{T}}{1-e^{-\frac{\hbar \omega-e V}{k_{B} T}}},
$$

and is displayed in Fig. 4. Notice that the antisymmetric part of this noise is the same as that of the ordinary resistor, $S_{V}(+\omega)-S_{V}(-\omega)=2 \hbar \omega R_{T}$, and is independent of the voltage. Also shown in Fig. 5 is the polarization and relaxation time, $\mathrm{T} 1$, of a $\mathrm{CPB}$ coupled to a shot noise environment. We see that full polarization is achieved only when $\hbar \omega_{01} \gg \mathrm{eV}$. For low transition frequencies, the polarization is inversely proportional to the current through the junction. Aguado and Kouwenhoven [11] have described the use of a double quantum dot as a two-level system to probe this behavior of the shot noise.

Given our discussion so far, it is now interesting to ask what the effects of a real quantum measurement on a quantum circuit will be. A quantum measurement device will in general be neither linear, Ohmic, nor equilib- 


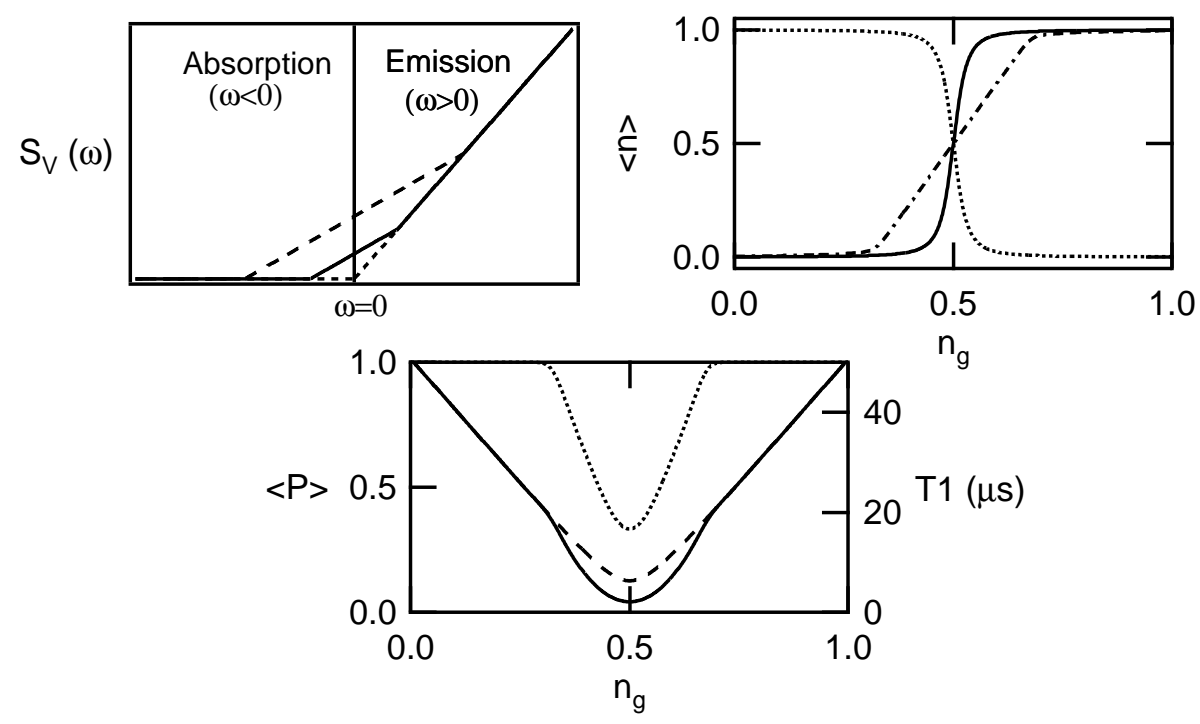

Figure 4. The box coupled to an nonequilibrium, Ohmic environment, i.e. a $50 \Omega$ tunnel junction. a) Two-sided noise spectral density, of the voltage, $S_{V}(\omega)$, for a junction at $\mathrm{T}=0.02 \mathrm{~K}$, with zero voltage (dotted line), and increasing bias voltages (solid and dashed lines). b) Average charge of box with $E_{C}=1 K, E_{J}=0.5 K$ when coupled with strength $\kappa=0.01$ to a junction biased at $e V=1.5 \mathrm{~K}$. c) Polarization (dotted line) and relaxation time $\mathrm{T} 1$ for the same parameters (solid) and for $\mathrm{T}=0, \mathrm{~V}=0$ (dashed line).

rium. Obviously, if we hope to characterize this measurement process, and to understand what one will observed when the qubit is coupled to the noise processes of the measuring device, we will need to calculate the full quantum (two-sided!) noise spectrum of the amplifier or detector.

\section{Single-Electron Transistor Coupled to a Two-Level System}

We have seen in previous sections that a two-level system (TLS) may be used as a "spectrum analyzer" to measure quantum noise. Here, we use this technique to theoretically calculate quantum noise. Instead of simply studying the "noisy" system of interest in isolation, one can study a composite system consisting of the "noisy" system coupled to a TLS; by calculating the relaxation and excitation rates of the TLS, one can efficiently calculate the quantum noise of interest ${ }^{9}$. We demonstrate the usefulness of this

\footnotetext{
${ }^{9}$ Note that the spirit of our approach is similar to that employed in the theory of full counting statistics [12]. There too one attaches an auxiliary spin $1 / 2$ to the scattering system of interest, and studies the dynamics of the fully coupled system to obtain the
} 
technique by outlining a calculation of the quantum charge noise of a single electron transistor (SET). This is an important example, as when an SET is used as an electrometer, it is this noise which determines the measurement backaction.

The SET consists of a metallic island attached via tunnel junctions to source and drain reservoirs. It is described by the Hamiltonian:

$$
\begin{aligned}
H_{S E T} & =\sum_{k, \alpha=L, R, I}\left(\varepsilon_{k}-\mu_{\alpha}\right) c_{k \alpha}^{\dagger} c_{k \alpha}+E_{C}(n-\mathcal{N})^{2}+H_{T} \\
H_{T} & =t \sum_{k, q, \alpha=L, R}\left(F^{\dagger} c_{k I}^{\dagger} c_{q \alpha}+\text { h.c. }\right)
\end{aligned}
$$

The first term in $H_{S E T}$ describes the kinetic energy of electrons in the leads $(\alpha=L, R)$ and on the island $(\alpha=I)$. The second term is the Coulomb charging energy which depends on $n$, the number of excess electrons on the island. This interaction term can be tuned by changing the voltage on a nearby gate electrode which is capacitively coupled to the island; $\mathcal{N}$ represents the dimensionless value of this voltage. Finally, $H_{T}$ describes the tunneling of electrons through the two SET tunnel junctions; the conductance of each junction (in units of $e^{2} / h$ ) is given by $g=4 \pi^{2} t^{2} \nu_{0}^{2}$, with $\nu_{0}$ being the density of states. $F^{\dagger}$ is an auxiliary operator which increases $n$ by one: $\left[n, F^{\dagger}\right]=F^{\dagger}$. For simplicity, we assume that the two junctions of the SET are completely symmetric (i.e. equal junction capacitances and dimensionless conductances).

Throughout this section, we will be interested in the regime of sequential tunneling in the SET, where transport involves energy-conserving transitions between two charge states of the SET island, say $n=0$ and $n=1$. These transitions are described by simple rates, which can be derived via Fermi's Golden rule:

$$
\begin{aligned}
\Gamma_{n \pm 1, n}^{\alpha} & =\gamma\left([\Delta E]_{n \pm 1, n}^{\alpha}\right) \\
\gamma(\Delta E) & =\frac{g \Delta E / h}{1-e^{-\Delta E /\left(k_{B} T\right)}} \\
\Delta E_{n \pm 1, n}^{\alpha} & =\mp 2 E_{C}\left(n \pm \frac{1}{2}-\mathcal{N}\right) \pm\left(\frac{1}{2}-\delta_{\alpha, R}\right) e V_{D S}
\end{aligned}
$$

$\Gamma_{n \pm 1, n}^{\alpha}$ is the tunneling rate from the charge state $n$ to $n \pm 1$ through junction $\alpha ; \Delta E$ is the energy gained in making the tunneling transition, and includes contributions both from the drain-source voltage $V_{D S}$ and from the charging energy. Sequential tunneling is the dominant transport mechanism when the junction conductances are small (i.e. $g /(2 \pi) \ll 1)$,

statistics of charge transfer in the scatterer.

rjsdraft12.tex; 28/05/2018; 5:41; p.19 
and the dimensionless gate voltage $\mathcal{N}$ is not too far away from a charge degeneracy point. Sequential tunneling is the most important regime for measurement applications, as it yields the largest SET currents.

At low temperatures, only tunnel events which follow the voltage are possible. There are thus there only two relevant rates: $n=0 \rightarrow 1$ transitions occur through the left junction at a rate $\Gamma_{10}^{L}$, while $n=1 \rightarrow 0$ occur through the right junction at a rate $\Gamma_{01}^{R}$. The average current will be given by:

$$
\langle I\rangle=e \bar{\Gamma} \equiv e \frac{\Gamma_{10}^{L} \Gamma_{01}^{R}}{\Gamma_{10}^{L}+\Gamma_{01}^{R}}
$$

We are interested in calculating $S_{Q}(\omega)$, the quantum noise associated with fluctuations of the charge on the central island of the SET. It is defined as:

$$
S_{Q}(\omega)=\int_{-\infty}^{\infty} d t\langle n(t) n(0)\rangle e^{-i \omega t}
$$

Note that we can equivalently think of $S_{Q}$ as describing the voltage noise of the island, as $V_{\text {island }}=e n / C_{\Sigma}$, where $C_{\Sigma}$ is the total capacitance of the island. In two limits, the form of the island charge noise can be anticipated. For $\omega \rightarrow 0$, the noise will correspond to classical telegraph noise- the island charge $n$ fluctuates between the values 0 and 1 , with Poisson-distributed waiting times determined by the rates $\Gamma_{10}^{L}$ and $\Gamma_{01}^{R}$. We thus expect a symmetric, Lorentzian form [13] for the noise at low frequencies:

$$
S_{Q}(\omega) \rightarrow \frac{2 \bar{\Gamma}}{\omega^{2}+\left(\Gamma_{10}^{L}+\Gamma_{01}^{R}\right)^{2}} \quad\left(\omega \ll E_{C}\right)
$$

For large frequencies $|\omega| \gg E_{C}$, we expect that correlations due to the charging energy will have no influence on the noise. The system will effectively look like two tunnel junctions in parallel, and we can use the results of Sec. 6 for the corresponding voltage noise. Noting that each junction effectively consists of a resistor and capacitor in parallel, we have at zero temperature:

$$
\begin{aligned}
S_{Q}(\omega) & =\frac{C_{\Sigma}^{2}}{e^{2}} \times S_{V}(\omega) \rightarrow \frac{C_{\Sigma}^{2}}{e^{2}}\left[2 \hbar \omega \operatorname{Re} Z_{t o t} \Theta(\omega)\right] \quad\left(|\omega| \gg E_{C}\right) \\
& =4\left(\frac{g}{2 \pi}\right) \frac{\omega}{\left(\frac{g}{2 \pi} \frac{4 E_{C}}{\hbar}\right)^{2}+\omega^{2}} \Theta(\omega)
\end{aligned}
$$

Note that $S_{Q}(\omega)$ decays as $1 / \omega$ at large frequencies, whereas Eq. (54) for classical telegraph noise decays as $1 / \omega^{2}$.

Given these two limiting forms, the question now becomes one of how the SET interpolates between them. One might expect that the two results 
should simply be added in quadrature, but as with combining thermal and quantum noise (see Sec. 6), this is approach is too simple. A completely quantum mechanical way of calculating the noise for any frequency is needed. This was recently accomplished by Johansson et. al [14] using an extension of a technique developed by Schöller and Schön [15]. Here, we rederive their results using the coupled system approach outlined above. This method is physically motivated and allows for a heuristic interpretation of the final result.

\section{SET Coupled to a Qubit}

We now consider a system where the SET is coupled to a two-level system (i.e. a qubit), with a coupling Hamiltonian which can induce transitions in the TLS. Using spin operators to describe the qubit, and assuming operation at the degeneracy point for simplicity, where the transitions are fastest ${ }^{10}$, we have:

$$
H=H_{S E T}-\frac{1}{2} \Omega \sigma_{x}+A \sigma_{z} n,
$$

where $\Omega$ is the qubit splitting frequency ${ }^{11}$, and $A$ is the coupling strength. We can define the rates $\Gamma_{\uparrow}$ and $\Gamma_{\downarrow}$ which are, respectively, the rate at which the qubit is excited by the SET, and the rate at which the qubit is relaxed by the SET. For a weak coupling $(A \rightarrow 0)$, one has (c.f. Eq. 14,13 in Sec. 2):

$$
\Gamma_{\downarrow / \uparrow}=\frac{A^{2}}{\hbar} S_{Q}( \pm \Omega)
$$

Eq. (57) tells us that if we know the rates $\Gamma_{\uparrow}$ and $\Gamma_{\downarrow}$ for a weakly coupled system at an arbitrary splitting frequency $\Omega$, we know the quantum noise $S_{Q}(\Omega)$ at all frequencies. This is the essence of the technique previously described, in which a qubit acts as a quantum spectrum analyzer of noise. Here, we mimic this approach theoretically by obtaining $\Gamma_{\uparrow}$ and $\Gamma_{\downarrow}$ from a direct analysis of the coupled system in the limit of weak coupling $(A \rightarrow 0)$. The object of interest is the reduced density matrix $\rho$ which describes both the charge $n$ of the transistor island and the state of the qubit. We are interested in two quantities. First, what is the stationary state of the qubit? The stationary populations of the two qubit states (which are determined

\footnotetext{
10 This amounts to maximizing the "destruction" due to the SET's noise, and the case where $\theta=\pi / 2$, the qubit eigenstates are in the $\sigma_{z}^{\prime}=\sigma_{x}$ direction, and the SET's perturbation is in the $-\sigma_{x}^{\prime}=\sigma_{z}$ direction (c.f. Eq. 42). After the noise of the SET is found, we can then recalculate the effects on the qubit at various $n_{g}$ or values of $\theta$ by including the modified matrix elements in the coupling coefficient, $A$.

11 Henceforth we use $\Omega$ for the transition frequency, instead of the previous notation $\omega_{01}=E_{01} / \hbar$, for compactness.
} 
from the time-independent solution for $\rho$ ) will tell us the polarization of the qubit, and the amount of asymmetry in the noise (c.f. Eq.40). Second, how quickly do the qubit populations relax to their stationary value? This relaxation will be described by a time-dependent solution of $\rho$ characterized by a mixing rate $\Gamma_{m i x}$ which is the sum of $\Gamma_{\uparrow}$ and $\Gamma_{\downarrow}$ (c.f. Eq. 41). From these two results we can solve for the individual values of $\Gamma_{\uparrow / \downarrow}$.

To deal with the dynamics of $\rho$, we make use of the fact that sequential tunneling processes are completely described by lowest-order perturbation theory in the tunneling Hamiltonian $H_{T}$. Keeping only second order terms (there are no non-vanishing first order terms), one obtains the following standard evolution equation in the interaction picture:

$$
\frac{d}{d t} \rho(t)=-\frac{1}{\hbar} \int_{-\infty}^{t} d t^{\prime}\left\langle\left[H_{T}(t),\left[H_{T}\left(t^{\prime}\right), \rho\left(t^{\prime}\right) \otimes \rho_{F}\right]\right]\right\rangle
$$

The angular brackets denote the trace over the single-particle degrees of freedom in the SET leads and island; $\rho_{F}$ is the equilibrium density matrix corresponding to the state of these degrees of freedom in the absence of tunneling. ${ }^{12}$ Note that a similar density matrix analysis of a qubit coupled to a SET was recently discussed by Makhlin et. al [16]; unlike the present case, these authors restricted attention to a vanishingly small splitting frequency $\Omega$.

To make progress with Eq. (58), we make a Markov approximation, which involves replacing $\rho\left(t^{\prime}\right)$ on the right-hand side with $\rho(t)$. This is permissible as we are interested in the slow dynamics of $\rho$. We want to find both the stationary solution of $\rho$, for which the Markov approximation is exact, and the mixing mode, a mode whose time dependence is $\propto e^{-\left(\Gamma_{\uparrow}+\Gamma_{\downarrow}\right) t}$. This mode is also arbitrarily slow in the weak coupling limit $A \rightarrow 0$ of interest. Finding the stationary mode and the mixing mode correspond to evaluating the polarization and T1 of the qubit (c.f. Eq. 40 and Eq. 41), as was shown earlier for the master equation of the probabilities in Section 5 . Note that the Markov approximation should be made in the Schrödinger picture, as it is in the Schrödinger picture that $\rho$ will be nearly stationary (i.e. all oscillations associated with the qubit splitting frequency $\Omega$ will be damped out in the long-time limit).

Evaluation of Eq. (58) in the Markov approximation results in the appearance of rates which are generalizations of those given in Eq. (49). Now, however, these rates depend on the initial and final state of the qubittunneling transitions can simultaneously change both the charge state of the SET island and the state of the qubit. The resulting equation is most

\footnotetext{
12 In the diagrammatic language of Ref. [15], Eq. (58) is equivalent to keeping all $\left(H_{T}\right)^{2}$ terms in the self-energy of the Keldysh propagator governing the evolution of $\rho$.
} 
easily presented if we write the reduced density matrix $\rho$ in the basis of eigenstates at zero tunneling. For each value of island charge $n$, there is a different qubit Hamiltonian, and correspondingly a different a qubit ground state $\left|g_{n}\right\rangle$ and excited state $\left|e_{n}\right\rangle$. When a tunneling event occurs in the SET, there is a sudden change in the qubit Hamiltonian. As the qubit ground and excited states at different values of $n$ are not orthogonal, tunneling transitions in the SET are able to cause "shake-up" transitions in the qubit. In the limit $A \rightarrow 0$, the relevant matrix overlaps are given by:

$$
\begin{aligned}
& \left\langle g_{m} \mid g_{n}\right\rangle=1-\frac{1}{2}\left(\frac{A(m-n)}{\Omega}\right)^{2}=\left\langle e_{m} \mid e_{n}\right\rangle \\
& \left\langle e_{m} \mid g_{n}\right\rangle=\frac{A(m-n)}{\Omega}
\end{aligned}
$$

Defining the frequency dependent rate $\Gamma_{n \pm 1, n}(\omega)$ as:

$$
\Gamma_{n \pm 1, n}(\omega) \equiv \sum_{\alpha=L, R} \gamma\left([\Delta E]_{n \pm 1, n}^{\alpha}+\hbar \omega\right),
$$

where $\Delta E$ and $\gamma(\Delta E)$ are defined in Eqs. (51) and (50), the required tunnel rates take the form:

$$
\Gamma_{m, n} \equiv \Gamma_{m, n}(0) \quad \Gamma_{m, n}^{ \pm} \equiv \Gamma_{m, n}( \pm \Omega)
$$

The $\Gamma^{+}$rates correspond to tunneling events where the qubit is simultaneously relaxed, and thus there is an additional energy $\Omega$ available for tunneling. For large $\Omega$, tunneling processes which are normally energetically forbidden can occur if they are accompanied by qubit relaxation. Similarly, the $\Gamma^{-}$rates describe tunnelling events where the qubit is simultaneously excited, with the consequence that there is less energy available for tunneling.

Returning to the evolution equation Eq. (58), note that we do not need to track elements of $\rho$ which are off-diagonal in the island charge index $n$ - there is no coherence between different charge states, as tunneling events necessarily create an electron-hole excitation. Further, if we focus on small qubit frequencies, we may continue to restrict attention to only $n=0$ and $n=1$ (i.e. $\Omega$ is not large enough to "turn on" tunneling processes which are normally energetically forbidden). Thus, there are 8 relevant matrix elements of $\rho$ - for each of the four qubit density matrix elements (i.e. $g g$, ee, ge, eg), there are two possible island charge states. We combine these elements into a vector $\vec{\rho}=\left(\rho_{g g}, \rho_{e e}, \rho_{g e}, \rho_{e g}\right)$, where $\rho_{g g}=\left(\left\langle 0, g_{0}|\rho| 0, g_{0}\right\rangle,\left\langle 1, g_{1}|\rho| 1, g_{1}\right\rangle\right)$, etc. Organizing the resulting evolution equation in powers of the coupling $A$, we obtain in the Schrödinger picture:

$$
\frac{d}{d t} \vec{\rho}=\left(\boldsymbol{\Lambda}_{\mathbf{0}}+\frac{A}{\Omega} \boldsymbol{\Lambda}_{\mathbf{1}}+\frac{A^{2}}{\Omega^{2}} \boldsymbol{\Lambda}_{\mathbf{2}}+\ldots\right) \vec{\rho}
$$


We discuss the significance of the matrices $\boldsymbol{\Lambda}_{\mathbf{j}}$ in what follows.

The $8 \times 8$ matrix $\boldsymbol{\Lambda}_{\mathbf{0}}$ describes the evolution of the system at zero coupling:

$$
\mathbf{\Lambda}_{\mathbf{0}}=\left(\begin{array}{cccc}
M & 0 & 0 & 0 \\
0 & M & 0 & 0 \\
0 & 0 & +i \Omega+M^{\prime} & 0 \\
0 & 0 & 0 & -i \Omega+M^{\prime}
\end{array}\right)
$$

with the $2 \times 2$ matrices $M$ and $M^{\prime}$ being defined by:

$$
M=\left(\begin{array}{cc}
-\Gamma_{10} & \Gamma_{01} \\
\Gamma_{10} & -\Gamma_{01}
\end{array}\right) \quad M^{\prime}=\frac{1}{2}\left(\begin{array}{cc}
-\left(\Gamma_{10}^{+}+\Gamma_{10}^{-}\right) & \Gamma_{01}^{+}+\Gamma_{01}^{-} \\
\Gamma_{10}^{+}+\Gamma_{10}^{-} & -\left(\Gamma_{01}^{+}+\Gamma_{01}^{-}\right)
\end{array}\right)
$$

At zero coupling there are no transitions between different qubit states, and hence $\boldsymbol{\Lambda}_{\mathbf{0}}$ has a block-diagonal form. There are two independent stationary solutions of Eq. (63) at $A=0$ (i.e. two zero eigenvectors of $\boldsymbol{\Lambda}_{\mathbf{0}}$ ), which correspond to being either in the qubit ground or qubit excited state:

$$
\vec{z}_{g}=\left(p_{0}, p_{1}, 0,0,0,0,0,0\right), \quad \vec{z}_{e}=\left(0,0, p_{0}, p_{1}, 0,0,0,0\right) .
$$

$\left(p_{0}, p_{1}\right)$ are the stationary probabilities of being in the $n=0$ or $n=1$ charge states:

$$
\left(p_{0}, p_{1}\right)=\left(\frac{\Gamma_{01}}{\Gamma_{01}+\Gamma_{10}}, \frac{\Gamma_{10}}{\Gamma_{01}+\Gamma_{10}}\right)
$$

The existence of two zero-modes is directly related to the fact that at zero coupling $(A=0)$, the probabilities to be in the qubit ground and excited state are individually conserved.

At non-zero coupling, the matrices $\boldsymbol{\Lambda}_{\mathbf{1}}$ and $\boldsymbol{\Lambda}_{\mathbf{2}}$ appearing in Eq. (63) generate transitions between different qubit states. The matrix $\boldsymbol{\Lambda}_{\mathbf{2}}$ directly couples $\rho_{g g}$ and $\rho_{e e}$, while $\boldsymbol{\Lambda}_{\mathbf{1}}$ couples $\rho_{g g}$ and $\rho_{e e}$ to the off-diagonal blocks $\rho_{g e}$ and $\rho_{e g}$. The effect of these matrices will be to break the degeneracy of the two zero modes of Eq. (63) existing at $A=0$. After this degeneracy is broken, there will still be one zero mode $\rho_{0}$, describing the stationary state of the coupled system (the existence of a stationary solution is guaranteed by the conservation of probability). For weak coupling, the qubit density matrix obtained from $\rho_{0}$ will be diagonal in the basis $\left\{\left|g_{\langle n\rangle}\right\rangle,\left|e_{\langle n\rangle}\right\rangle\right\}$, which corresponds to the average SET charge $\langle n\rangle=p_{1}$. The ratio of the occupancies of these two qubit states will yield the ratio between the relaxation rate $\Gamma_{\downarrow}$ and the excitation rate $\Gamma_{\uparrow}$. In addition, there will also be a slow, time-dependent mode of Eq. (63) arising from breaking the degeneracy of the two $A=0$ zero modes. This time-dependent mode will describe how a linear combination of $z_{g}$ and $z_{e}$ relaxes to the true stationary state, and will have an eigenvalue $\lambda=-\Gamma_{\uparrow}-\Gamma_{\downarrow}$, i.e. the mixing rate. 
Thus, we need to do degenerate second order perturbation theory in the coupling $A$ to obtain the relaxation and excitation rates $\Gamma_{\downarrow}$ and $\Gamma_{\uparrow}$. The only subtlety here is that the matrix $M$ is not Hermitian, implying that it has distinct right and left eigenvectors. Letting $\overrightarrow{\tilde{z}}$ represent the left eigenvector of $\boldsymbol{\Lambda}_{\mathbf{0}}$ corresponding to the right eigenvector $\vec{z}$, we define the projector matrix $\mathbf{P}$ as:

$$
\mathbf{P}=\left|z_{g}\right\rangle\left\langle\tilde{z}_{g}|+| z_{e}\right\rangle\left\langle\tilde{z}_{e}\right|,
$$

and let $\mathbf{P}_{\perp}$ denote $1-\mathbf{P}$. As usual, degenerate second order perturbation theory requires diagonalizing the perturbation in the space of the degenerate eigenvectors. We are thus led to look at the matrix $Q$, defined as:

$$
Q=\frac{A^{2}}{\Omega^{2}}\left(\mathbf{P} \boldsymbol{\Lambda}_{\mathbf{2}} \mathbf{P}+\mathbf{P} \boldsymbol{\Lambda}_{\mathbf{1}} \mathbf{P}_{\perp}\left[-\boldsymbol{\Lambda}_{\mathbf{0}}\right]^{-\mathbf{1}} \mathbf{P}_{\perp} \boldsymbol{\Lambda}_{\mathbf{1}} \mathbf{P}\right)
$$

¿From the definition of Q, we may immediately identify the rates $\Gamma_{\uparrow}$ and $\Gamma_{\downarrow}:$

$$
\Gamma_{\uparrow}=\left\langle\tilde{z}_{e}|Q| z_{g}\right\rangle \quad \Gamma_{\downarrow}=\left\langle\tilde{z}_{g}|Q| z_{e}\right\rangle
$$

We thus see how the rates $\Gamma_{\uparrow, \downarrow}$ arise in the present approach- they are related to breaking the degeneracy between two zero-modes (stationary solutions) which exist at zero coupling. Note that there are two distinct contributions to $\Gamma_{\uparrow, \downarrow}$, coming from the two terms in the matrix $Q$ : a "direct" contribution involving $\boldsymbol{\Lambda}_{\mathbf{2}}$ and an "interference" contribution involving $\boldsymbol{\Lambda}_{\mathbf{1}}$ acting twice. These two terms have a different physical interpretation, as will become clear.

Let us first consider the rate $\Gamma_{\uparrow}$, which describes how noise in the SET causes ground to excited state transitions in the qubit. For this rate, our approximation of only keeping two charge states will be valid for all splitting frequencies $\Omega$. To evaluate the "direct" contribution to this rate, which involves the first term in the matrix $Q$, note that the relevant part of $\boldsymbol{\Lambda}_{\mathbf{2}}$ has the expected form:

$$
\left.\mathbf{\Lambda}_{\mathbf{2}}\right|_{e e, g g}=\left(\begin{array}{cc}
0 & \Gamma_{01}^{-} \\
\Gamma_{10}^{-} & 0
\end{array}\right)
$$

i.e. it consists of tunnel rates which correspond to having given up an energy $\Omega$ to the qubit. Using Eqs. (69) and (70), we find:

$$
\left.\Gamma_{\uparrow}\right|_{\text {direct }}=\left(\frac{A}{\Omega}\right)^{2}\left(p_{0} \Gamma_{10}^{-}+p_{1} \Gamma_{01}^{-}\right)=\left(\frac{A}{\Omega}\right)^{2}\left(\frac{\Gamma_{01} \Gamma_{10}^{-}+\Gamma_{10} \Gamma_{01}^{-}}{\Gamma_{10}+\Gamma_{01}}\right)
$$

The direct contribution to $\Gamma_{\uparrow}$ has a very simple form: for each charge state $n=0,1$, add the rate to tunnel out of $n$ while exciting the qubit, weighted 
by both the probability to be in state $n$, and the overlap between ground and excited states (i.e. $\left.(A / \Omega)^{2}\right)$. This is very similar to how one typically calculates the current for a SET: one adds up the current associated with each charge state (i.e. a difference of rates), weighted by the occupancy of the state. The direct contribution to $\Gamma_{\uparrow}$ neglects any possible coherence between successive excitation events; as a result, it fails to recover the classical expression of Eq. (54) in the small- $\Omega$ limit.

We now consider the "interference" contribution to $\Gamma_{\uparrow}$ coming from the second term in the expression for matrix $Q$ (c.f. Eq. (69)). After some algebra, we obtain the following for the interference contribution to $\Gamma_{\uparrow}$ :

$$
\left.\Gamma_{\uparrow}\right|_{i n t}=-\frac{2 A^{2}}{\Omega^{2}}\left(p_{0} \Gamma_{10}^{-}+p_{1} \Gamma_{01}^{-}\right) \frac{\left(\Gamma_{\Sigma}\right)^{2}}{\Omega^{2}+\left(\Gamma_{\Sigma}\right)^{2}}
$$

where:

$$
\Gamma_{\Sigma} \equiv \frac{\Gamma_{10}^{-}+\Gamma_{10}^{+}+\Gamma_{01}^{-}+\Gamma_{01}^{+}}{2}
$$

This contribution is purely negative, and is only significant (relative to the direct contribution) at low frequencies $\Omega<\Gamma$. We can interpret this equation as describing the interference between two consecutive excitation events. For example, consider the first term in Eq. (73). This describes a process where a SET initially in the charge state $n=0$ undergoes a tunnel event to the $n=1$ state, creating a superposition of qubit ground and excited states. At some later time the SET relaxes to the stationary distribution $\left(p_{0}, p_{1}\right)$ of the charge states, again partially exciting the qubit. Letting $\Delta t$ represent the time between these two events, we have the approximate sequence:

$$
\begin{aligned}
\left|0, g_{0}\right\rangle & \stackrel{\Gamma_{10}^{-}}{\longrightarrow}\left|1, g_{1}\right\rangle+\alpha\left|1, e_{1}\right\rangle \\
& \stackrel{\Delta t}{\longrightarrow} e^{i \Omega \Delta t / 2}\left|1, g_{1}\right\rangle+e^{-i \Omega \Delta t / 2} \alpha\left|1, e_{1}\right\rangle \\
& \stackrel{\Gamma_{\Sigma}}{\longrightarrow}\left(e^{i \Omega \Delta t / 2} \beta-e^{-i \Omega \Delta t / 2} \alpha\right)\left|0, e_{0}\right\rangle+\ldots
\end{aligned}
$$

Here, $\alpha$ is the amplitude associated with qubit excitation having occurred during the first $(n=0 \rightarrow 1)$ tunnel event, while $\beta$ is the amplitude associated with excitation occurring during the second $(n=1 \rightarrow 0)$ tunneling. These amplitudes will be given by the corresponding matrix overlap elements:

$$
\alpha=\left\langle e_{1} \mid g_{0}\right\rangle \simeq \frac{A}{\Omega} \quad \beta=\left\langle e_{0} \mid g_{1}\right\rangle \simeq-\frac{A}{\Omega}
$$

In the final state after the two tunnelings (Eq. (76)), there are two terms in the amplitude of the state $\left|0, e_{0}\right\rangle$, corresponding to the fact that qubit excitation could have occurred in either the first or the second tunnel event. 
To get a rate for this double excitation event, we should take the modulus squared of the final $\left|0, e_{0}\right\rangle$ state amplitude, then multiply by the occupancy of the initial state $\left(p_{0}\right)$ and the rate of the first tunnel event $\left(\Gamma_{10}\right)$. The interference term in the resulting expression takes the form:

$$
\left.\Gamma_{\uparrow}\right|_{\text {int }}=\left(p_{0} \Gamma_{10}\right) \times 2 \operatorname{Re}\left(\alpha^{*} \beta e^{i \Omega \Delta t}\right)=-\left(p_{0} \Gamma_{10}\right) \frac{2 A^{2}}{\Omega^{2}} \cos (\Omega \Delta t)
$$

The above expression is a function of the time $\Delta t$ between the first and second tunnel events. This time is determined by the fact that the intermediate superposition state (Eq. (75)) corresponds to a non-stationary distribution of charge on the SET island, and will decay via tunneling to the stationary distribution $\left(p_{0}, p_{1}\right)$ at a rate $\Gamma_{\Sigma}$. Taking this decay to be Poissonian, and averaging over $\Delta t$, we obtain:

$$
\left.\Gamma_{\uparrow}\right|_{i n t}=-\left(p_{0} \Gamma_{10}\right) \frac{2 A^{2}}{\Omega^{2}} \frac{\left(\Gamma_{\Sigma}\right)^{2}}{\Omega^{2}+\left(\Gamma_{\Sigma}\right)^{2}}
$$

This is precisely the first term in Eq. (73); the second term can be obtained in the same way, by now considering a situation where the SET is initially in the $n=1$ charge state. As claimed, $\left.\Gamma_{\uparrow}\right|_{\text {int }}$ corresponds to the interference between two consecutive excitation events. The negative sign of this contribution can be directly traced to the matrix overlap elements (c.f. Eq. 77). Also, we see that the suppression of the interference term at large $\Omega$ results from phase randomization occurring during the delay time between the two excitation events.

Returning to the total noise, we combine Eq. (73) with the direct contribution Eq. (72) to $\Gamma_{\uparrow}$; comparing against Eq. (57), we obtain the final expression for $S_{Q}(\Omega)$ at all negative frequencies:

$$
S_{Q}(-|\Omega|)=\frac{p_{0} \Gamma_{10}^{-}+p_{1} \Gamma_{01}^{-}}{\Omega^{2}+\frac{1}{4}\left(\Gamma_{10}^{-}+\Gamma_{10}^{+}+\Gamma_{01}^{-}+\Gamma_{01}^{+}\right)^{2}}
$$

Note for large $|\Omega|$ (i.e. $\left.|\Omega|>\max \left(\Delta E_{01}^{\alpha}, \Delta E_{10}^{\alpha}\right) \simeq V_{D S} / 2\right), S_{Q}(-\Omega)$ will vanish identically at zero temperature. Physically, this cutoff corresponds to the largest amount of energy the SET can give up to the qubit during a single tunnel event; giving up more energy would suppress the event completely (i.e. the tunnel rates have a step-function form at zero temperature, c.f. Eq. (50)). If one included higher order processes in the tunneling (i.e. went beyond sequential tunneling), correlated tunneling events involving the full voltage drop over both junctions, $V_{D S}$, would move this cutoff to higher values of absolute frequency.

We now turn to the relaxation rate $\Gamma_{\downarrow}$, and hence the positive frequency parts of $S_{Q}$. The calculation proceeds exactly as that for $\Gamma_{\uparrow}$, the only 


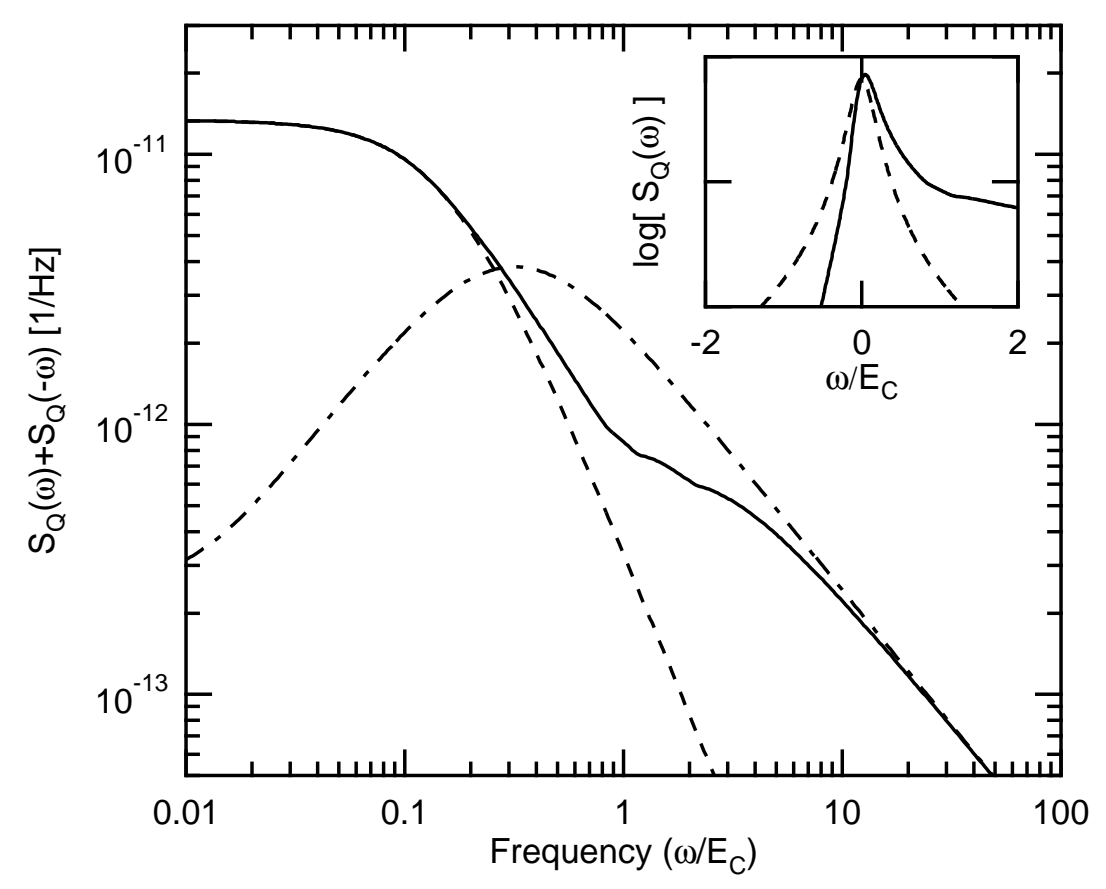

Figure 5. Symmetrized SET charge noise as a function of frequency, for typical SET parameters: $g=1, E_{C} / k_{B}=2 \mathrm{~K}, \mathcal{N}=0.33, e V_{D S}=E_{C}$, and $T=20 \mathrm{~m} K$. The dashed line is the classical telegraph noise (Eq. (54)), while the dot-dashed line is the noise of two parallel tunnel junctions (Eq. (55)). Inset: full (non-symmetrized) quantum noise for identical SET parameters; the dashed line is the symmetric classical telegraph noise.

modification being that one now needs to include the charge states $n=2$ and $n=-1$, as the SET could absorb enough energy from the qubit to make transitions to these states possible. We can combine the result for $\Gamma_{\downarrow}$ with Eq. (80) to obtain a single, compact expression for the noise at all frequencies first obtained by Johansson et. al [14]: ${ }^{13}$

$$
S_{Q}(\omega)=\frac{p_{0}\left[\Gamma_{10}(\omega)+\Gamma_{-1,0}(\omega)\right]+p_{1}\left[\Gamma_{01}(\omega)+\Gamma_{21}(\omega)\right]}{\omega^{2}+\frac{1}{4}\left[\Gamma_{10}(\omega)+\Gamma_{10}(-\omega)+\Gamma_{01}(\omega)+\Gamma_{01}(-\omega)\right]^{2}}
$$

Shown in Figure 5 is the symmetrized noise $S_{Q}(\omega)+S_{Q}(-\omega)$ for typical SET parameters. One can clearly see abrupt changes in the slope of this curve; each of these kinks corresponds to a threshold frequency at which a given tunneling process either turns on or turns off. For comparison, curves

13 Eq. (81) ignores additional order $g /(2 \pi)$ terms which arise in the denominator at positive frequencies large enough to turn on tunneling to higher charge states; such terms are clearly negligible in the sequential tunneling regime due to the smallness of $g /(2 \pi)$. 

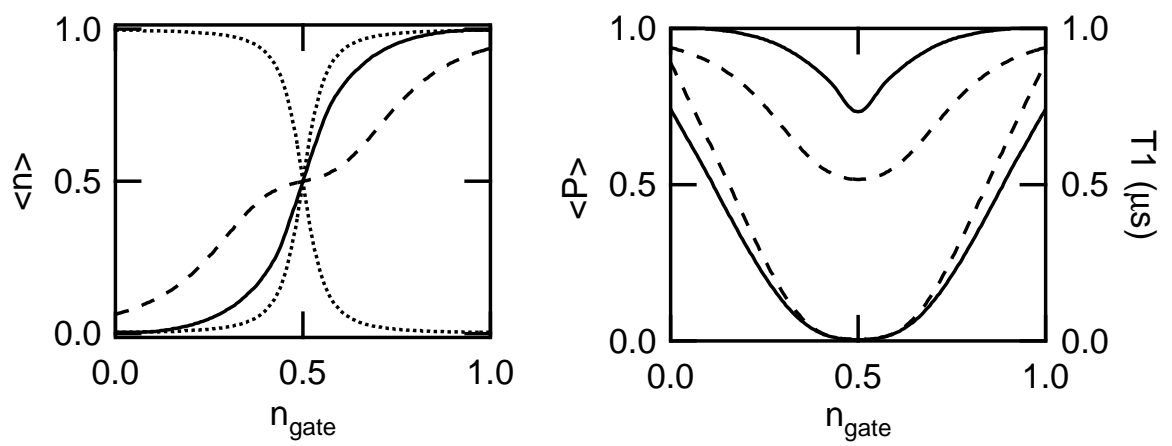

Figure 6. a) Average charge state of a Cooper pair box coupled to a SET, as a function of box gate voltage, using identical SET parameters as above. The box parameters are $E_{C} / k_{B}=0.5 \mathrm{~K}, E_{J} / k_{B}=0.25 \mathrm{~K}$ and the coupling constant is $\kappa=0.04$. We also include relaxation effects due to a $10 \%$ coupling to a $50 \Omega$ environment. The dashed curve corresponds to assuming the SET produces classical telegraph noise, the solid curve corresponds to using the full quantum noise of the SET, and the dashed-dot curve is the box ground state. b) The relaxation time $T_{1}$ for the same system, as a function of box gate voltage.

corresponding to classical telegraph noise and to the uncorrelated noise of two tunnel junctions are also shown. At low frequencies the symmetrized true noise matches the classical curve; for higher frequencies, it lies above the classical curve but below the curve corresponding to the uncorrelated case. The inset of this figure shows the both the negative and positive frequency parts of $S_{Q}(\omega)$.

It is easy to check that in the limit $\omega \rightarrow 0$, Eq. (81) recovers the classical telegraph expression of Eq. (54). In the high-frequency, zero temperature limit, one can also see that Eq. (81) approaches the uncorrelated result of Eq. (55) from below:

$$
S_{Q}(\omega) \rightarrow \Theta(\omega) \frac{4\left(\frac{g}{2 \pi}\right) \omega\left(1-\frac{E_{C}}{2 \hbar \omega}\right)}{\omega^{2}+\frac{g^{2}}{\pi^{2}} \omega^{2}} \rightarrow \Theta(\omega) \frac{2 g}{\pi \omega}
$$

Note that at high frequencies, it is only the "direct" terms which contribute to the noise- the interference contribution is not important in the limit of uncorrelated tunneling. The fact that the noise approaches the high frequency limit from below results from the tendency of charging energy induced correlations (which are present for a finite $\omega / E_{C}$ ) to suppress fluctuations of $n$, and thus suppress the noise. Note that the interpolation between the low and high frequency limits here is very different than, e.g., interpolating between thermal noise and zero-point fluctuation noise in a tunnel junction. In the latter case, one is effectively combining two sources 
of noise; here, one is simply turning off correlations brought on by the charging energy by increasing $\omega$.

Finally, shown in Figure 6 is the average charge state of a Cooper pair box coupled to a SET with identical parameters to that in Fig. 5. We have also included here the relaxation effects of the environment, modelled as in Sec. 6 as a $50 \Omega$ impedance. Note that even near the box degeneracy point, there are large deviations between the result obtained using the full quantum noise of the SET and that obtained from using only classical telegraph noise. In Fig. 6b, we show the relaxation rate $T_{1}$ for the same system. Note that the differences between using the full quantum noise and the classical expression are not so evident here.

\section{Summary}

In this article, we have emphasized the need to discuss quantum noise processes using their two-sided spectral densities. Because of the quantum nature of noise, the positive and negative frequencies are generally unequal, in order to account for spontaneous emission. A two-level system was shown to be an ideal spectrum analyzer for probing the quantum nature of a noise process or reservoir. With the advent of real electrical circuits which behave as coherent two-level systems (e.g., [5],[7]), we can now build and use quantum electrical spectrum analyzers. We also described the use of a qubit as a theoretical tool, by following the evolution of the density matrix of a TLS coupled to the noise-producing system of interest. This technique appears to be quite powerful, as it can yield analytical results for the full quantum noise spectrum of a wide variety of devices, including the superconducting SET [17]. The distinction between the classical noise and the quantum noise, found in this way, leads to dramatically different predictions (c.f. Fig. 6 and Ref. [17]) for continuous measurements of qubits with an SET. The "coupled-system" calculational approach also allows predictions of the dephasing by the measurement, the performance relative to the Heisenberg uncertainty limit [13], the fidelity of single-shot measurements of the qubit states, and the effects of strong coupling to the qubit. The combined theoretical and experimental advances raise many interesting possibilities for testing our understanding of quantum measurement theory with mesoscopic devices.

\section{Acknowledgements}

The authors acknowledge the generous support of this work by the NSA and ARDA under ARO contracts ARO-43387-PH-QC (RS,SG) and DAAD1902-1-044 (MD), by the NSF under DMR-0196503 \& DMR-0084501 (AC,SG), 
the David and Lucile Packard Foundation (RS), and the W.M. Keck Foundation.

\section{References}

1. A.O. Caldeira and A.J. Leggett, Ann. Phys. 149, 374 (1983).

2. H.B. Callen and T.A. Welton, Phys. Rev. 83, 34 (1951).

3. M.H. Devoret, in Quantum Fluctuations, S. Reynaud, E. Giacobino and J. ZinnJustin, eds. (Elsevier, Amsterdam, 1995).

4. V. Bouchiat, D. Vion, P. Joyez, D. Esteve and M. Devoret, Physica Scripta T76, 165 (1998).

5. D. Vion, A. Aassime, A. Cottet, P. Joyez, H. Pothier, C. Urbina, D. Esteve, and M.H. Devoret, Science 296, 886 (2002).

6. J.M. Martinis et al., submitted to Phys. Rev. B, 2002.

7. K.W. Lehnert, K. Bladh, L.F. Spietz, D. Gunnarson, D.I. Schuster, P. Delsing, and R.J. Schoelkopf, submitted to Phys. Rev. Lett., 2002.

8. V.B. Braginsky and F. Ya. Khalili, "Quantum Measurement," (Cambridge University Press, New York, 1992).)

9. A.J. Dahm et al., Phys. Rev. Lett. 22, 1416 (1969); D. Rogovin and D.J. Scalapino, Ann. Phys. 86, 1 (1974).

10. R.J. Schoelkopf, P.J. Burke, A.A. Kozhevnikov, D.E. Prober, and M.J. Rooks, Phys. Rev. Lett. 78, 3370 (1997).

11. R. Aguado and L.P. Kouwenhoven, Phys. Rev. Lett. 84, 1986 (2000).

12. L. S. Levitov, H. Lee and G. B. Lesovik, J. Math. Phys. 37, 4845 (1996).

13. M.H. Devoret and R.J. Schoelkopf, Nature 406, 1039 (2000).

14. G. Johansson, Andreas Käck, and Göran Wendin, Phys. Rev. Lett. 88, 046802 (2002).

15. H. Schoeller and G. Schön, Phys. Rev. B, 18436 (1994).

16. Y. Makhlin, B. Shnirman and G. Schön, Phys. Rev. Lett. 85, 4578 (2000); ibid., Rev. Mod. Phys. 73, 357 (2001).

17. A. A. Clerk, S. M. Girvin, A. K. Nguyen and A. D. Stone, Phys. Rev. Lett. 89, 176804 (2002). 